\title{
Influence du climat sur l'expression des composantes de la croissance en hauteur chez le pin noir d'Autriche (Pinus nigra Arn. ssp nigricans)
}

\author{
J.P. GUYON \\ avec Ia collaboration technique de D. BETORED \\ INRA, Station de Sylviculture méditerranéenne. \\ Avenue A. Vivaldi, F 84000 Avignon
}

\begin{abstract}
Résumé
Après avoir étudié l'importance de chacune des composantes d'un cycle morphogénétique de pin noir d'Autriche (Pinus Nigra Arn. ssp nigricans), sur la valeur finale de l'accroissement annuel en hautcur, on a cherché à relier l'expression de ces composantes à des paramètres elimatiques simples (précipitations et température).

L'analyse du nombre d'entré-nouds présents sur les 6 dernières années (1979 ì 1984) ainsi que les mesures de l'élongation des entre-nœuds pendant les trois dernières saisons de végétation (1982 à 1984) mettent en évidence que l'élaboration d'une pousse annuelle est essenticllement conditionnée par la phase d'initiation des entre-nœuds.

Les paramètres climatiques explicatifs de la croissance annuelle en hautcur sont alors :

- les précipitations cumulées des mois de juin, juillet et septembre d'une année donnée qui expliquent au mieux d'une part le nombre d'entre-nœuds initiés cette année là et d'autre part l'accroissement total en hauteur de l'annće suivante;

- les températures minimales des mois d'avril et mai dont dépend l'importance de l'allongement moyen de ces entre-nœuds.

Mots clés : Accroissement hauteur, morphogénèse, cycle morphogénétique, taux de croissance, température, pluviométrie, Pinus nigra, initiation des euphylles, élongation des entre-nouds.
\end{abstract}

\section{Introduction}

La croissance en hauteur est le principal critère dendrométrique utilisé lors de la construction des modèles de croissance. L'étude des différents facteurs climatiques qui interviennent dans l'expression de ce caractère est donc un élément majeur préalable à la prédiction et à l'explication de la vigueur des arbres et des peuplements.

La pousse totale annuelle de la plupart des conifères monocycliques à croissance fixe résulte de l'expression de deux composantes qui sont d'une part le nombre d'entre-nœuds initiés une année donnée et d'autre part, l'allongement, l'année suivante, de ces entre-nœuds (fig. 1). 


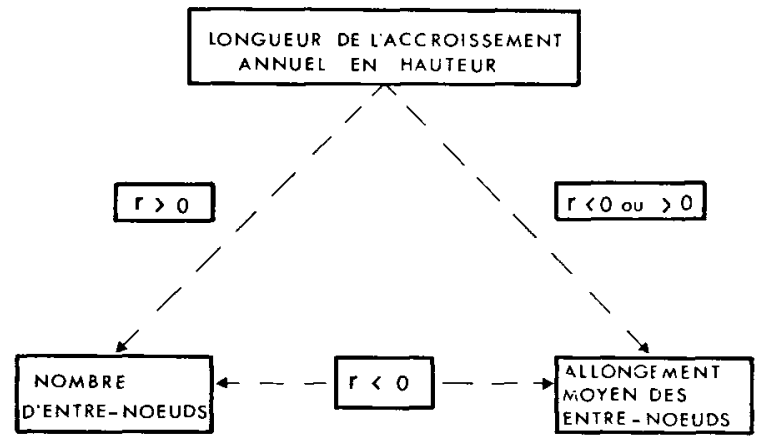

FIG. 1

Types de corrélations généralement observés entre les composantes de la croissance en hauteur.

Components of annual height increment and correlations between those parameters.

Les études récentes sur les composantes de la croissance en hauteur chez quelques conifères : Cannell (1978), Kremer (1981), Kremer \& Roussel (1982), Lascoux (1984), ont toutes mis en évidence un modèle de croissance qui, tout en étant fortement déterminé par des facteurs génétiques n'en est pas moins instable dans le temps (fig. 2).

Cette instabilité dans le temps est attribuée par certains de ces auteurs, à une forte interaction génotype $X$ année.

Les deux paramètres climatiques influant généralement sur la croissance en hauteur sont le facteur hydrique et le facteur thermique.

L'influence d'un stress hydrique, au cours des mois de juin et juillet, sur l'initiation des entre-nœuds a été mise en évidence, expérimentalement, par GarretT \& Zahner (1973) et Pollard \& Logan (1977). Enfin Owens (1984) lors d'une étude sur Abies grandis a montré que la période d'initiation se situait en juin-juillet mais qu'elle pouvait se poursuivre au-delà du mois d'août, jusqu'en novembre.

Le facteur température de l'air peut limiter l'initiation des entre-nouds (KREMER \& LARSON, 1983) sur Pinus banksiana ainsi que l'élongation de ces entre-nœuds (LANNER, 1968).

Notre travail sur le pin noir se propose, à partir dobservations sur six années, de déterminer d'une part l'importance de chacune des composantes de la croissance en hauteur et d'autre part l'influence de paramètres climatiques simples (précipitations et température) sur l'accroissement annuel en hauteur.

\section{Matériel et méthodes}

Les mesures ont été effectuées dans la parcelle 20 de la «série des Cèdres» de la Forêt Communale de Bédoin (Vaucluse). Cette parcelle, située à $900 \mathrm{~m}$ d'altitude, sur la face sud du Mont Ventoux a étć parcourue, en partie, par un incendie en 1973. Sur 1 ha environ, s'est installée dès le printemps 1974 une régénération 
NOMBRE

D'ENTRE - NOEUDS

ACCROISSEMENT

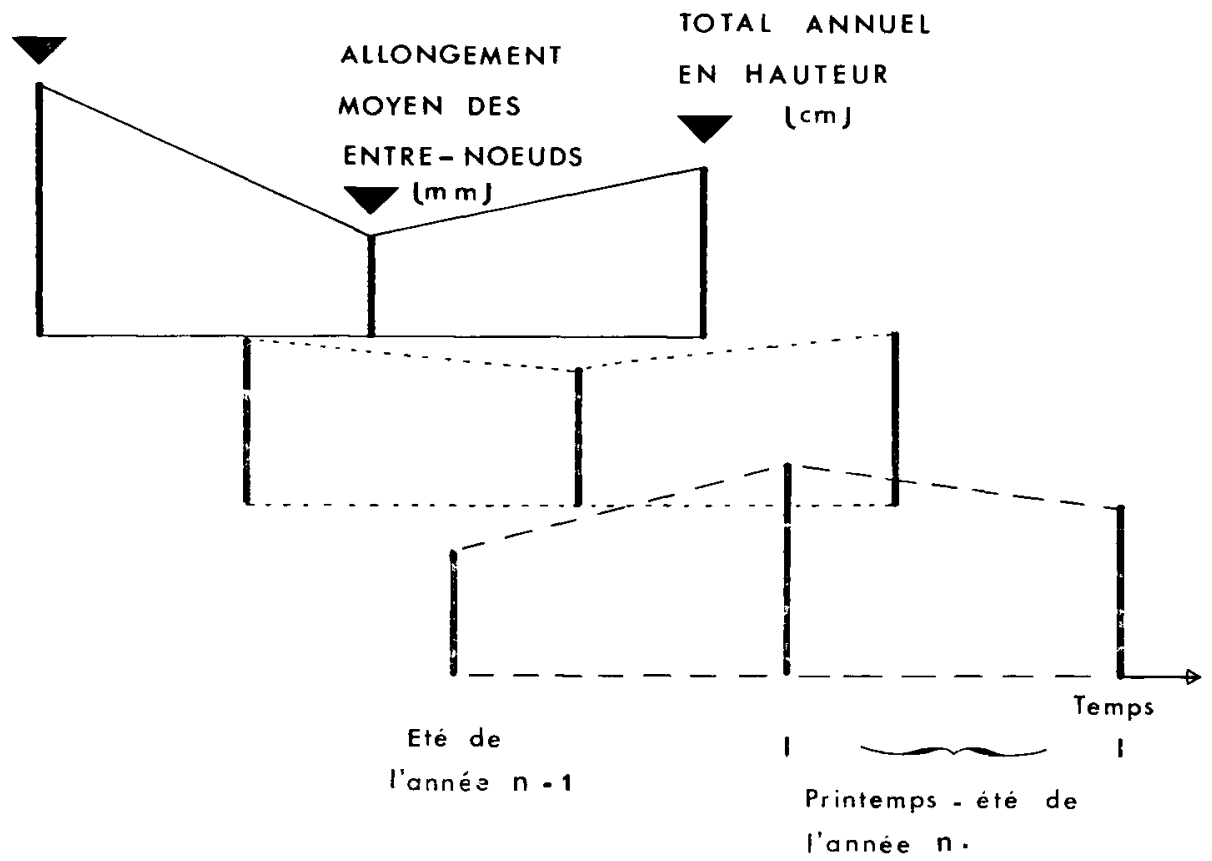

Fig. 2

Exemple de 3 types de croissance pouvant donner le même accroissement en hauteur. An exemple of different growth patterns producing the same annual shoot growth.

mélangée de cèdre, pin noir et pin sylvestre. A cette date, 90 individus de pins noirs ont été repérés pour une étude sur la croissance aérienne (J. ToTH, 1984). Les mesures concernant la présente étude ont été effectuées sur un sous-échantillon de 30 individus choisis de façon aléatoire parmi les 90 individus primitivement retenus.

\subsection{Les différents types de mesures}

\subsection{L'accroissement total annuel en hauteur}

Chaque année, la longueur de la pousse terminale a été mesurée à la fin de la période de végétation. La mesure a été faite avec la précision du centimètre, de la base de la pousse à l'apex du bourgeon terminal.

\subsection{L'élongation périodique}

Sur 3 ans $(1982,1983,1984)$, l’allongement de la pousse terminale a été mesuréc au millimètre près à raison :

- d'une mesure tous les quinze jours en 1982 ; 
- d'une mesure tous les quinze jours puis toutes les semaines en 1983 ;

- d'une mesure hebdomadaire en 1984.

Enfin du 31-8-1982 au 3-3-1983 et du 27-8-1983 au 6-3-1984, seule la longueur du bourgeon a été relevée, respectivement à 7 et 11 reprises.

\subsection{Le dénombrement et l'allongement des entre-nouds}

A chaque entre-nueud correspond un point végétatif. Les points végétatifs, situés à la base des euphylles, peuvent évoluer de 3 façons différentes suivant leur position sur la pousse (E.F. Drbazac, 1963), (fig. 3) :

- à la base, ils ne se développent pas du tout, d'où le nom d'écaille «stérile» donné aux euphylles de cette partie de la pousse ;

- au-dessus de cette partie stérile, ils donnent naissance à des rameaux courts ou brachyblastes qui porteront les pseudophylles (ou aiguilles);

- au sommet, ils évoluent en cônelets et en bourgeons subterminaux. L'allongement de ces bourgeons produira des rameaux longs latéraux.

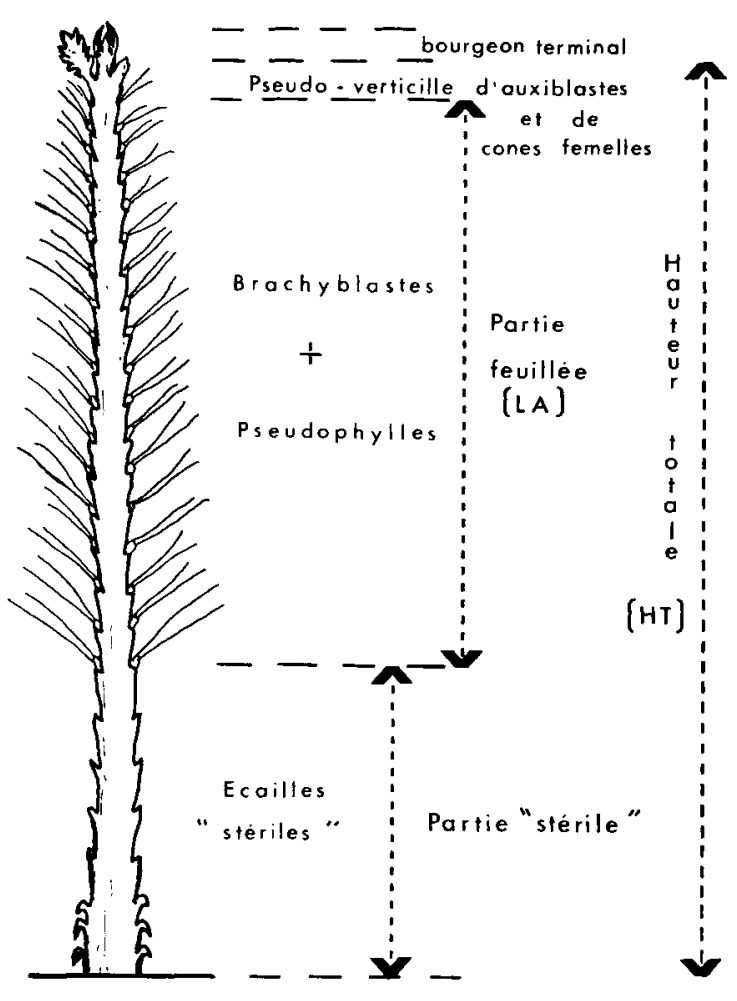

FIG. 3

Description d'un cycle morphogénétique (d'après KREMER \& RousseL, 1982).

Description of a morphogenetic cycle (in KREMFR \& Roussel, 1982). 
Le nombre total de brachyblastes, de branches et de cônes a donc été noté par comptage direct sur les pousses terminales des années 1979 à 1984 selon la méthode décrite par A. Kremer \& G. Roussel (1982) (fig. 4). En ce qui concerne les écailles stériles, leur nombre n'a pu être relevé de façon fiable que pour les 4 dernières années.

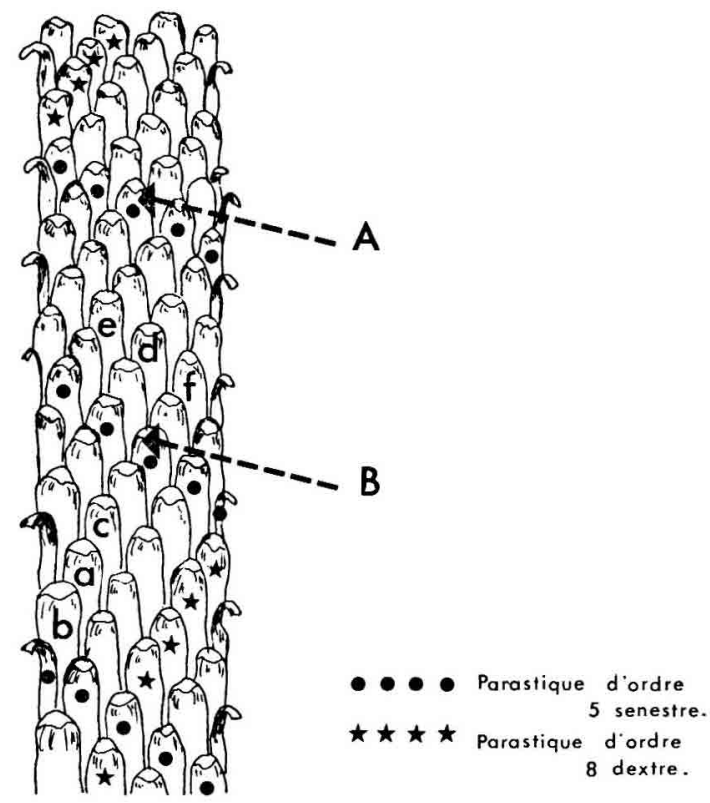

FIG. 4

Description d'une partie de la pousse annuelle (d'après KREMER \& RousSEL, 1982).

Description of a segment of the annual shoot. Phyllotactic arrangements of the cataphylls (in KREMER \& Roussel, 1982).

Les points A et B sont les intersections d'un parastique d'ordre 5 aves 1 . verticale passant au niveau du tronc et sont séparés de $5 \mathrm{n}$ entre-nœuds où $\mathrm{n}$ est le nombre d'entre-nceuds sur une révolution du parastique donné. Sur ce schéma ne sont représentées que les euphylles ou cataphylles : les structures axillaires (brachyblastes, auxiblastes ou cônelets) n'ont pas été dessinées. La longueur moyenne d'un entre-nœud (LME) sur cette partie est alors : LME $=\frac{\text { distance de A à B }(\mathrm{mm})}{5 \mathrm{n}}$ (d'après Kremer \& Roussel, 1982).

La longueur moyenne des entre-nœuds a été évaluée :

- à 3 niveaux de la pousse feuillée : à la base, au milieu et au sommet;

- sur la totalité de la longueur de la pousse feuillée (LA). La longueur moyenne des entre-nœuds (LME), dans ce cas, a pour expression : LA/NE où NE représente le nombre d'entre-nouds présents sur la partie feuillée. 


\subsection{Les paramètres climatiques}

Les données climatiques relatives aux années 1979 à 1984 proviennent, à l'exception des températures de 1984, d'un poste météorologique équipé d'un thermohygrographe, d'un pluviographe et d'un pluviomètre, installé depuis 1973 à un kilomètre environ de la placette d'étude. Les températures minimales de l'année 1984 sont celles relevées d'un poste climatique installé au centre du dispositif au début de cette année-là.

\subsection{L'analyse des données}

\subsection{Composantes de la croissance en hauteur}

La longueur de l'accroissement de la pousse feuillée (LA) une année donnée est le résultat du produit du nombre d'entre-nœuds (NE) par la longueur moyenne des entre-nœuds (LME) soit :

(1) $\mathrm{LA}=\mathrm{NE} \times \mathrm{LME}$ et :

(2) $\log (\mathrm{LA})=\log (\mathrm{NE})+\log (\mathrm{LME})$ soit, en termes de variances :

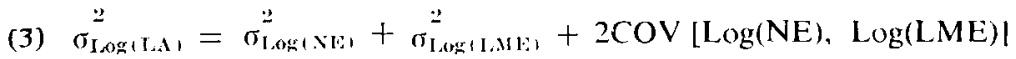

en divisant chacun des termes par $\sigma_{\text {Lort LA }}^{2}$, il vient :

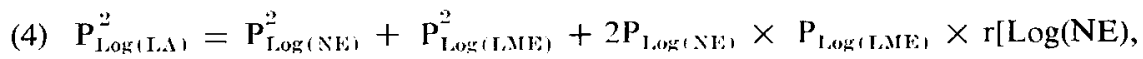
$\log (\mathrm{LME})]$

où

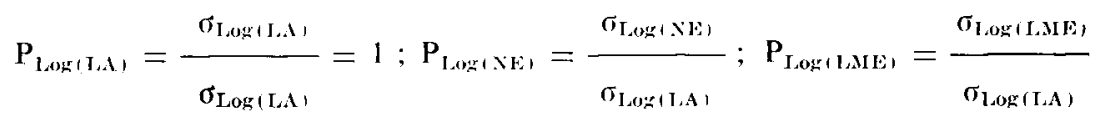

et $r[\log (N E), \log (L M E)]$ est le coefficient de corrélation entre $\log (N E)$ et $\log (\operatorname{LME})$.

Kremer \& LARSON (1983) proposent une représentation vectorielle de l'équation (4) (cf. fig. 7) et il vient :

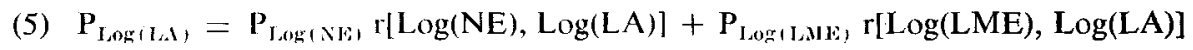

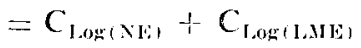

L'effet de chacune des composantes de la croissance en hauteur d'une pousse feuillée sur la longueur de l'accroissement est alors analysé au moyen des 3 paramètres suivants :

a) Le coefficient de piste $\left(P_{\mathrm{I}_{\text {og }}(\mathrm{NE})}\right.$ ou $\left.\mathrm{P}_{\mathrm{LaxilME}}\right)$ qui traduit la variabilité relative de chacune des composantes (NE et LME) dans la valeur finale (LA) de l'accroissement.

b) Le coefficient de corrélation r qui rend compte des liaisons entre les différentes composantes.

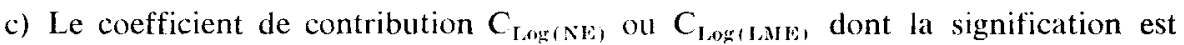
identique à celle du coefficient de piste mais qui tient compte de la liaison qui existe entre chacune des composantes et la longueur de l'accroissement. 


\subsection{L'élongation périodique de l'accroissement total en hauteur}

Les données recueillies par quinzaine ou par semaine ont été ajustées à une fonction logistique généralisće de Richards (Richards, 1959; Causton \& Vénus, 1981). Les paramètres de ces courbes de croissance permettent d'estimer des paramètres intéressants au plan biologique, à savoir :

a) La durée de l'élongation.

b) Le taux de croissance absolu moyen qui est le rapport de la valeur finale de laccroissement à la duréc de l'élongation.

Enfin, à partir des donnćes brutes, il a été calculé les taux de croissance absolus. Ces taux qui correspondent à la moyenne journalière de l'élongation calculée d'après nos relevés sont représentés sur la figure 5 pour les 3 années de mesure (1982 à 1984).

\subsection{Les relations climat-croissance en hauteur} dantes.

Elles ont été calculées au moyen de régressions progressives multiples ascen-

\section{Résultats}

\subsection{L'élongation annuelle}

L’analyse des résultats fait apparaître :

a) que la durée d'élongation et le taux de croissance interviennent de la même façon sur la valeur finale de l'accroissement (tabl. 1) ;

b) que les taux de croissance maximum sont observés immédiatement après le premier maximum de la température minimale moyenne (fig. 5);

c) que plus des $2 / 3$ de l'accroissement est réalisé en six semaines (fig. 5);

\section{TABleaU 1}

Caractéristiques de l'élongation annuelle estimées par la fonction de Richards.

Parameters of shoot growth curves estimated by the Richard's function for 3 years.

\begin{tabular}{c|c|c|c|c}
\hline Année & $\begin{array}{c}\text { Durée } \\
\text { de l'élongation } \\
\text { (Jour) }\end{array}$ & $\begin{array}{c}\text { Taux de croissance } \\
\text { absolu maximum } \\
(\mathrm{mm} / \mathrm{J})\end{array}$ & $\begin{array}{c}\text { Taux de croissance } \\
\text { absolu moyen } \\
(\mathrm{mm} / \mathrm{J})\end{array}$ & $\begin{array}{c}\text { Valeur finale } \\
\text { de l'asymptote* } \\
(\mathrm{mm})\end{array}$ \\
\hline & 52 & 10,66 & 6,65 & \\
1982 & $\ldots$ & 7,08 & 4,75 & 348,35 \\
1983 & $\ldots$ & 6,52 & 4,13 & 368,14 \\
1984 & 60 & 6,52 & 249,16 \\
\hline
\end{tabular}

* Bourgeon non compris. 


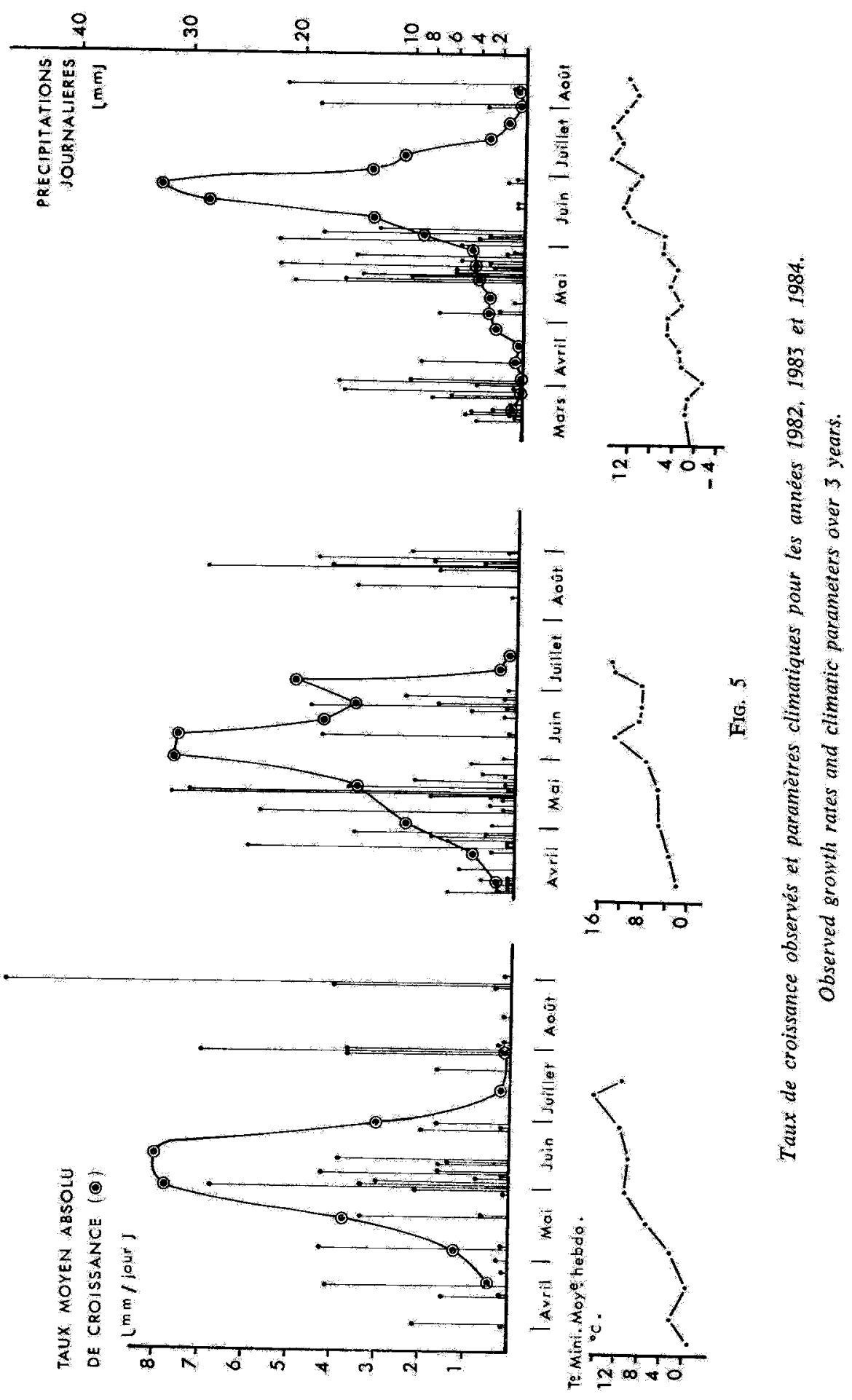


d) que l'allongement du bourgeon est réalisé, en grande partie, avant la fin du mois d'août (fig. 6). Cet allongement qui traduit la formation d'entre-nœuds (mais qui peut aussi traduire un allongement de la partie stérile), peut se poursuivre jusqu'en octobre comme ce fut le cas en 1982. Il est d'ailleurs remarquable que ce prolongement de l'allongement du bourgeon coïncide avec une pluviométrie plus. importante au mois de septembre 1982. L'essentiel du développement du bourgeon se réaliserait donc pendant les mois de juin et juillet.

Aucune corrélation significative n'est apparue entre le taux de croissance absolu sur une période (quinzaine ou semaine) et le total des précipitations de la période considérée. De même, il n'a pas été trouvé de relation (coefficient de corrélation = $-0,0120$ ) entre la pluviométrie cumulée de la période d'élongation (mars à juillet) et l'allongement total de la pousse.

Par contre, un coefficient de corrélation de 0,888 (significatif au seuil de 1 p. 100) a été trouvé entre le taux moyen de croissance absolu journalier observé sur une semaine et la température minimale de la semaine pour la période du 6-3-1984 au 26-6-1984, c'est-à-dire jusqu'à la période de croissance maximum. Au-delà de cette date, le parallélisme entre température et taux de croissance n'existe plus. En ce qui concerne les années 1982 et 1983, la fréquence des observations n'a pas permis. de trouver une corrélation aussi étroite qu'en 1984.

\subsection{Analyse des composantes de la croissance en hatteur}

Les principaux résultats sont regroupés dans le tableau 2.

L'essentiel de la pousse totale est constitué par l'accroissement de la partie feuillée. Les classements des moyennes des accroissements totaux annuels et des entre-nouds présents sur la tige feuillée sont identiques.

Aux deux années de forte croissance (1982 et 1983) correspond un grand nombre d'entre-nœuds. A l'inverse, les années où l'on a constaté les plus faibles. accroissements sont aussi celles où l'on dénombre le moins d'entre-nouds (1979 et 1980).

Pour les années intermédiaires (1981 et 1984), bien que les longueurs totales de l'accroissement ne soient pas significativement différentes, le nombre d'entrenoeuds de 1984 est supérieur à celui de 1981 ; la différence significative de l'allongement moyen des entre-næuds explique alors la similitude des accroissements totaux de ces deux années.

Les résultats de l'élongation des entre-nœuds sur différentes parties de la tige confirment ceux de KREMER \& Roussel (1982) à savoir la croissance plus importante des entre-nœuds au milieu de la pousse feuillée, cette croissance intervenant au moment où les conditions climatiques sont les plus favorables.

Les relations entre les composantes ainsi que l'importance de chacune des composantes sont précisćes au moyen des différents coefficients (tabl. 3). 
Tableau 2

Comparaison interannuelle des composantes de la croissance en hauteur. Comparison over 6 years of height growth components.

1) Accroissement des différentes parties de la pousse annuelle (mm) et comparaison des moyennes (Test Duncan).

\begin{tabular}{c|c|c|c|c|c}
\hline Année & $\begin{array}{c}\text { Partie stérile } \\
\text { seuil 1\% }\end{array}$ & Année & $\begin{array}{c}\text { Partie feuillée } \\
\text { seuil 1\% }\end{array}$ & Année & $\begin{array}{c}\text { Pousse totale (1) } \\
\text { seuil 1\% }\end{array}$ \\
\hline & 42,62 & 1983 & 327,17 & 1983 & 388,70 \\
1983 & 35,69 & 1982 & 320,31 & 1982 & 372,67 \\
1982 & 30,97 & 1984 & 229,89 & 1984 & 284,76 \\
1979 & 28,69 & 1981 & 225,93 & 1981 & 264,83 \\
1981 & 28,67 & 1980 & 195,33 & 1980 & 234,63 \\
1984 & 21,00 & 1979 & 163,77 & 1979 & 209,06 \\
1980 & & &
\end{tabular}

2) Accroissement des entre-nauds sur différentes parties de la tige feuillée (mm).

\begin{tabular}{c|c|c|c|c|c|c|c} 
Année & $\begin{array}{c}\text { Allongement } \\
\text { à la base } \\
\text { seuil 1 \% }\end{array}$ & Année & $\begin{array}{c}\text { Allongement } \\
\text { au milieu } \\
\text { seuil 1\% }\end{array}$ & Année & $\begin{array}{c}\text { Allongement } \\
\text { au sommet } \\
\text { seuil 1\% }\end{array}$ & Année & $\begin{array}{c}\text { Allongement } \\
\text { moyen } \\
\text { seuil 1\% }\end{array}$ \\
\hline 1981 & 1,30 & 1981 & 1,30 & 1981 & 1,07 & 1981 & 1,24 \\
1982 & 1,20 & 1982 & 1,23 & 1980 & 0,99 & 1983 & 1,18 \\
1983 & 1,16 & 1983 & 1,21 & 1982 & 0,96 & 1982 & 1,18 \\
1980 & 1,06 & 1980 & 1,18 & 1983 & 0,92 & 1980 & 1,15 \\
1984 & 0,96 & 1984 & 0,98 & 1979 & 0,76 & 1984 & 0,96 \\
\hline
\end{tabular}

3) Dénombrement des entre-næuds présents sur la pousse annuelle.

\begin{tabular}{c|c|c|c|c}
\hline Année & $\begin{array}{c}\text { Nombre } \\
\text { d'entre-nœuds } \\
\text { partie feuillée } \\
\text { seuil 1\% }\end{array}$ & $\begin{array}{c}\text { Nombre } \\
\text { de bourgeons } \\
\text { seuil 1\% }\end{array}$ & $\begin{array}{c}\text { Nombre d'écailles } \\
\text { stériles (2) } \\
\text { seuil 1\% }\end{array}$ & $\begin{array}{c}\text { Nombre total } \\
\text { d'entre-næuds (2) } \\
\text { seuil 1\% }\end{array}$ \\
\hline 1983 & 279,93 & 4,45 & 29,64 & 314,36 \\
1982 & 272,03 & 4,07 & 23,79 & 299,90 \\
1984 & 238,74 & 3,67 & 23,59 & 265,96 \\
1981 & 184,86 & 3,66 & 10,28 & 198,04 \\
1979 & 182,53 & 3,23 & - & - \\
1980 & 170,00 & & - &
\end{tabular}

(1) Bourgeon compris.

(2) Les mesures de 1979 et de 1980 n'étaient pas assez fiables pour être comptabilisées. 


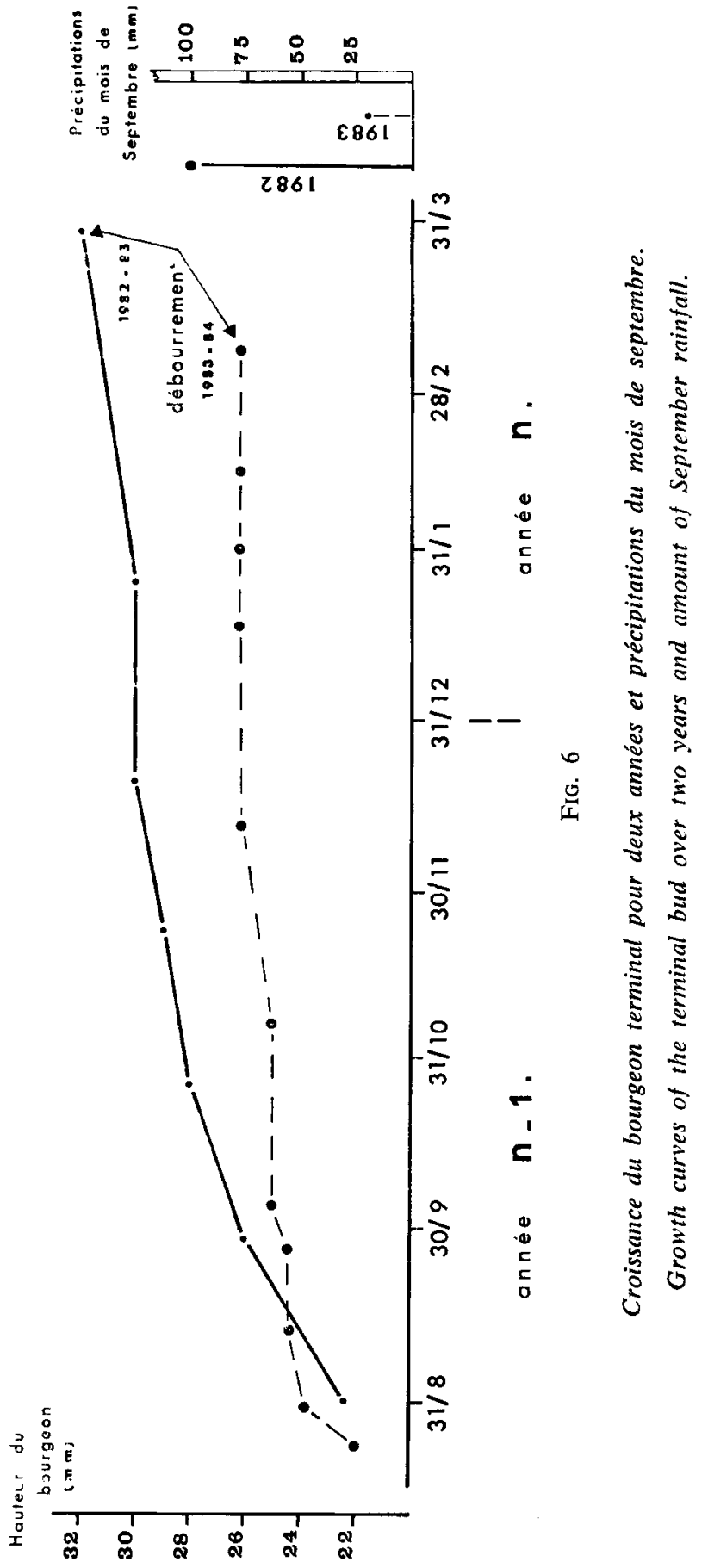


Tableau 3

Coefficients de pistes, de contribution et de corrélation pour les valeurs de Log NE et Log LME.

Variation of path, correlation and contribution coefficients of height growth components over 6 years.

\begin{tabular}{c|l|c|c|c|c}
\hline Année & $\begin{array}{c}\text { Compo- } \\
\text { santes }\end{array}$ & $\begin{array}{c}\text { Coefficient } \\
\text { de piste }\end{array}$ & $\begin{array}{c}\text { Coefficient } \\
\text { de contribution }\end{array}$ & $\begin{array}{c}\text { Correlation entre } \\
\text { LA et LME } \\
\text { ou LA et NE }\end{array}$ & $\begin{array}{c}\text { Correlation } \\
\text { entre LME } \\
\text { et NE }\end{array}$ \\
\hline 1979 & Log NE & 0,697 & 0,612 & $0,878^{* *}$ & $+0,316 \mathrm{NS}$ \\
& Log LME & 0,527 & 0,388 & $0,732^{* *}$ & \\
1980 & Log NE & 0,880 & 0,618 & $0,702^{* *}$ & $-0,229 \mathrm{NS}$ \\
& Log LME & 0,718 & 0,382 & $0,532^{* * *}$ & \\
1981 & Log NE & 0,752 & 0,463 & $0,615^{* *}$ & $-0,169 \mathrm{NS}$ \\
& Log LME & 0,798 & 0,537 & $0,671^{* *}$ & \\
1982 & Log NE & 0,774 & 0,590 & $0,763^{* *}$ & $-0,016 \mathrm{NS}$ \\
& Log LME & $\mathbf{0 , 6 4 7}$ & 0,410 & $0,634^{* *}$ & \\
1983 & Log NE & 0,884 & 0,640 & $0,723^{* *}$ & $-0,227 \mathrm{NS}$ \\
& Log LME & 0,710 & 0,360 & $0,508^{* *}$ & \\
1984 & Log NE & 0,771 & 0,664 & $0,863^{* *}$ & $-0,176 \mathrm{NS}$ \\
& Log LME & 0,520 & 0,336 & $0,649^{* *}$ & \\
Total & Log NE & 0,784 & 0,584 & $0,745^{* *}$ & $-0,062 \mathrm{NS}$ \\
\hline
\end{tabular}

*** : Significatif à 1 p. 100.

NS : Non significatif.

LA = longueur de la pousse feuillée.

$\mathrm{NE}=$ nombre d'entre-nœud de la partie feuillée.

LME = longueur moyenne d'un entre-noud de la partie feuillée.

Quelle que soit l'annće, une corrélation positive et significative existe d'une part entre LA et NE et d'autre part entre LA et LME.

A l'exception de l'année 1981, les coefficients de piste et de contribution de NE sont plus grands que ceux de LME, traduisant la part plus importante prise par la variation du nombre d'entre-nœuds dans la variation de la pousse terminale. Pour ce qui est de 1981, chacune des composantes apporte une contribution équivalente.

Ces résultats concordent avec ceux obtenus par A. Kremer \& G. Roussel (1982) et LASCOUX (1984) sur pin maritime.

Au plan biologique, des corrélations négatives entre NE et LME traduiraient un phénomène de «compensation» entre ces deux composantes: l'allongement des entre-nœuds pourrait être d'autant plus important que le nombre d'entre-nœuds initiés 

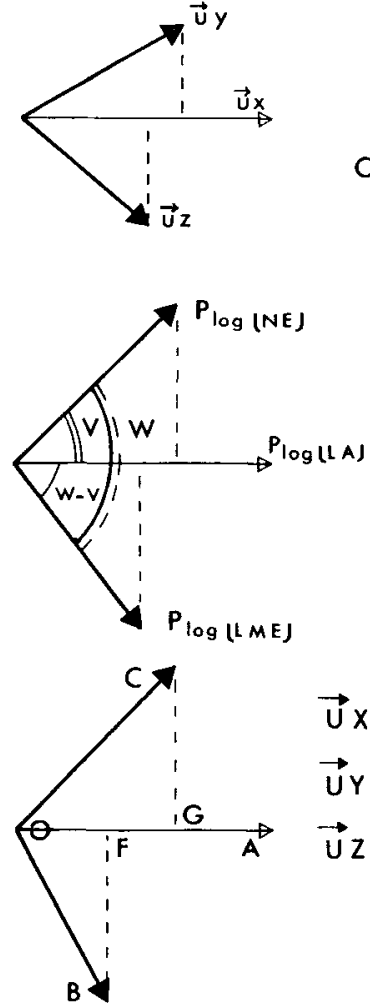

$\cos (W)=r_{\log (N E), \log (L M E)}$

$\cos (V)=r_{\log (N E), \log (L A)}$

$\cos (W-V)=r_{\log (N E), \log (L M E)}$
$P_{\log (L A)}=P_{\log (N E)} \cos |V|+$

$P_{\log (L M E)} \cos (W-V)$

$P_{\log (L A)}=C_{\log (N E)}+C_{\log (L M E)}$

$\vec{U} X$ vecteur unitaire de $\log |L A|$

$\vec{U} Y \quad " \quad \| \quad \log (N E)$

$\vec{U} Z \| \quad " \quad \log |L M E|$

FIG. 7

Représentation vectorielle du coefficient de piste de $\log (L A), \log (N E), \log (L M E)$.

Graphical representation of components analysis.

est faible. Ce phénomène de compensation n'apparaît pas aussi clairement dans nos résultats. En effet les corrélations entre ces deux composantes changent de signe d'une année sur l'autre et ne sont jamais significatives.

\subsection{Variables climatiques explicatives de la croissance en hauteur}

Compte tenu de nos précédentes observations, les variables climatiques retenues pour expliquer l'accroissement total annuel en hauteur $\left(\mathrm{H}_{\mathbf{T}}\right)$ (tabl. 4 et fig. 8) sont :

a) Les précipitations cumulées de juin, juillet et septembre de l'année précédente $(\mathrm{Pn}-1)$. Le calcul des coefficients de corrélation simple entre la hauteur de laccroissement l'année $\mathrm{n}$ et le total des précipitations de chacun des mois précédant cet accroissement fait apparaître que ce sont ces trois mois qui sont le mieux corrélés avec les accroissements totaux annuels. 
ANNEE $n-1$.

Température minimale moyenne de juin et Juillet $\left({ }^{\circ} \mathrm{C}\right)$.

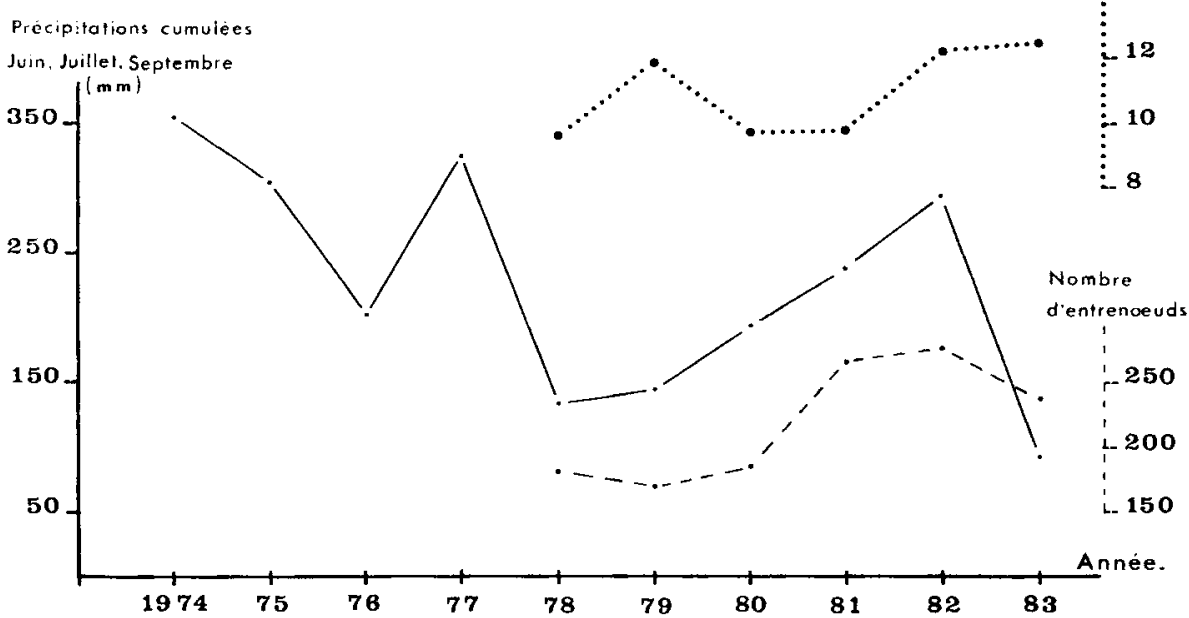

Hauteur de

laceroissement annuel

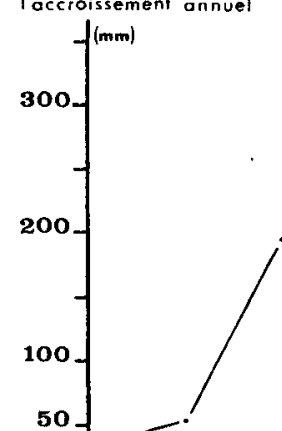

ANNEEN.

$$
\text { E } n
$$




\section{TABLEAU 4}

Coefficients de correlation entre les composantes de la croissance en hauteur et les paramètres climatiques. Equations des régressions multiples.

Correlation coefficients between height growth components and climatic parameters. Multiple regression analysis.

Equations de régressions multiples linéaires:

nombre de d.d.l.

$\mathrm{H}_{\mathrm{1}}=-201,4+1,17 \mathrm{NE}+147,7 \mathrm{LME}+0,32 \mathrm{Pn}-1$

$\left(\mathrm{R}=0,999^{* *}\right) \quad(3$ et 2$)$

$\mathrm{H}_{\mathrm{T}}=109,6+0,9464 \mathrm{Pn}-1$

$\left(\mathrm{R}=0,861^{*}\right) \quad(1$ et 4$)$

$\mathbf{H}_{\mathrm{T}}=19,9+15,07 \mathrm{Pn}-1+1,123 \mathrm{Tn}-1-29,23 \mathrm{Tn}$

$(\mathrm{R}=0,917 \mathrm{NS}) \quad(3$ et 2$)$

$\mathrm{NE}=332,3+0,66 \mathrm{Pn}-1-62,32 \mathrm{Tn}-1 \quad(\mathrm{R}=0,767 \mathrm{NS}) \quad(2$ et 3$)$

\begin{tabular}{|c|c|c|c|}
\hline \multirow[b]{2}{*}{ Facteurs climatiques } & \multicolumn{3}{|c|}{ Composantes de la croissance en hauteur } \\
\hline & $\begin{array}{l}\text { Hauteur de } \\
\text { l'accroissement } \\
\text { annuel }\left(H_{\bullet}\right)\end{array}$ & $\begin{array}{c}\text { Nombre } \\
\text { d'entre-nœuds (NE) } \\
\text { de la poussée } \\
\text { feuillée }\end{array}$ & $\begin{array}{c}\text { Allongement moyen } \\
\text { (LME) de la pousse } \\
\text { feuillée }\end{array}$ \\
\hline $\begin{array}{l}\text { Température minimale moyen- } \\
\text { ne des mois de juin et juillet } \\
\text { de l'année } n-1(\operatorname{Tn}-1)\end{array}$ & 一 & $0,270 \mathrm{NS}$ & 一 \\
\hline $\begin{array}{l}\text { Précipitations cumulées des } \\
\text { mois de juin, juillet et sep- } \\
\text { tembre de l'année } n-1 \\
(\text { Pn }-1) \ldots \ldots \ldots \ldots \ldots\end{array}$ & $0,861 *(1)$ & $\begin{array}{l}0,592 \text { NS }(1) \\
0,927 * * 2)\end{array}$ & 一 \\
\hline $\begin{array}{l}\text { Température minimale moyen- } \\
\text { ne des mois d'avril ct mai } \\
\text { de l'année } n(T n) \ldots \ldots \ldots\end{array}$ & $0,430 \mathrm{NS}(1)$ & 一 & $0,807 *(3)$ \\
\hline $\begin{array}{l}\text { : Significatif à } 5 \text { p. } 100 . \\
\text { NS : Non signiciatif à } 1 \text { p. } 100 . \\
\text { d.d.l. : Degré de libertifté. } \\
\text { (1) Coefficient calculé sur } 6 \text { ans } \\
\text { (2) Coefficient calculé pour la } \\
\text { (3) Coefficient calculé sur } 3 \text { ans }\end{array}$ & $\begin{array}{l}\text { iode 1980-1983. } \\
\text { 1981-1983). }\end{array}$ & & \\
\hline
\end{tabular}

Les coefficients de corrélation non significatifs sont de $0,443,0,405$ et 0,709 respectivement pour juin, juillet et septembre. En ce qui concerne le mois d'août, le coefficient de corrélation trouvé est de $-0,261$. Les précipitations orageuses qui arrivent régulièrement vers la mi-août tombent sur un sol sec et provoquent un important ruissellement. Les végétaux ne profitent donc que partiellement de ces pluies. L'initiation ralentit vers cette époque et peut se poursuivre jusqu'en décembre (comme ce fut le cas en 1982) si les conditions climatiques sont favorables. Ces différentes observations expliquent d'une part l'importance des pluies de septembre sur l'initiation et, d'autre part, l'absence de corrélation entre la croissance en hauteur d'une année et les précipitations du mois d'août de l'année précédente. 
b) La température minimale ( $\mathrm{Tn}$ ) des mois d'avril et mai, période de début et de milieu d'élongation.

c) La température minimale $(\mathrm{Tn}-1)$ des mois de juin et juillet de l'année précédente, période d'initiation des entre-nœuds.

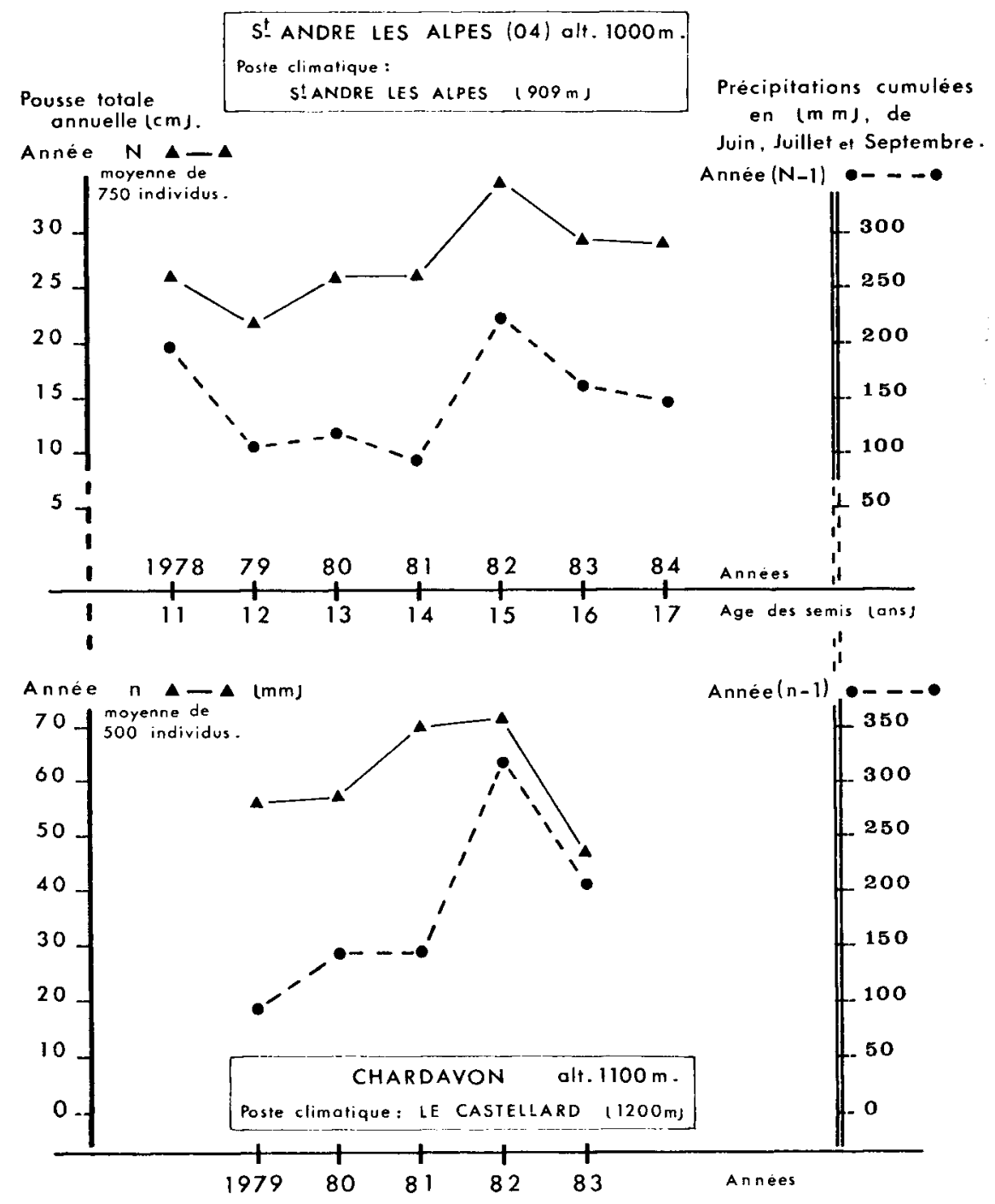

FIG. 9

Précipitations et croissance en hauteur sur 2 dispositifs des Alpes de Haute-Provence.

Variation of annual shoot length and cumulated rainfalls of June, July and September of the previous years at two forest sites. 
Les corrélations significatives obtenues entre $\mathrm{Pn}-1$ et $\mathrm{H}_{\mathrm{r}}, \mathrm{Pn}-1$ et le nombre d'entre-nœuds de la pousse feuillée NE, et enfin, Tn et la longueur moyenne des entrenœuds de la pousse feuillée LME, confirment d'une part l'importance des précipitations de l'année précédente dans la constitution de la pousse terminale et d'autre part l'influence de Tn sur LME.

La diminution du coefficient de corrélation entre $\mathrm{Pn}-1$ et NE quand on passe de 4 à 6 années d'observation ainsi que le fait que la corrélation sur 6 années calculée entre $\mathrm{Pn}-1$ et $\mathbf{H}_{\mathrm{T}}$ soit meilleure qu'entre $\mathrm{Pn}-1$ et $\mathrm{NE}$ incitent à formuler des hypothèses quant aux variations des potentialités d'initiation en fonction de l'âge. On peut en effet penser, compte tenu de l'allure générale des courbes de croissance en hauteur, que les potentialités d'initiation augmentant avec l'âge pendant la phase juvénile, un même niveau de pluviométrie n'aura pas le même effet sur des semis que sur des arbres plus âgés. Il apparaît donc que les faibles précipitations de 1983 n'ont pas été aussi fortement accusées par les arbres que celles de 1978 et 1979, ce qui expliquerait l'absence de signification du coefficient de corrélation quand on intègre ces 2 années dans le calcul de la régression.

Les équations de régression rassemblées dans le tableau 4 montrent que l'accroissement total en hauteur d'une année peut s'expliquer par la combinaison des 3 paramètres climatiques décrits plus haut. L'absence de signification des coefficients de corrélation multiple s'explique par le faible nombre des mesures dont nous disposons (6 années).

Le même type de relation entre $\mathrm{Pn}-1$ et $\mathrm{H}_{\mathrm{T}}$ a été également mis en évidence sur deux dispositifs d'étude de la régénération naturelle du pin noir situés dans les Alpes-de-Haute-Provence (fig. 9). Si la relation s'avère évidente pour Saint-Andréles-Alpes (régénération naturelle après incendie), elle est beaucoup moins nette pour Chardavon. Le lait que la régénération sur ce dernier dispositif ait été obtenue par coupes progressives (la coupe définitive ayant eu lieu en 1983) et l'éloignement du poste climatique peuvent expliquer dans ce cas l'absence de relations étroites entre $\mathrm{H}_{\mathrm{T}}$, et $\mathrm{Pn}-1$.

\section{Discussion et conclusion}

1

Les mesures et observations effectuées sur 6 ans permettent donc de préciser la part de chacune des composantes (NE et LME) dans la constitution de la pousse feuillée ainsi que les mécanismes de croissance en hauteur du pin noir d'Autriche en fonction des conditions climatiques, à savoir :

1) L'importance de NE dans la constitution d'une pousse feuillée et par conséquent de l'accroissement total en hauteur puisque celui-ci est en grande partie expliqué par la longueur de la tige feuillée.

En schématisant, le nombre d'entre-næuds contribue pour 60 p. 100 à la constitution de la pousse, l'allongement moyen des entre-nœuds contribue, lui, pour 40 p. 100. Cette répartition de la contribution diffère de celle obtenue sur pin maritime dans les Landes par Lascoux (1984) qui observe une contribution plus élevée du nombre d'entre-nouds. Cette différence pourrait s'expliquer par des effets plus marqués, en région méditerranéenne, de la sécheresse sur l'initiation des entre- 
nœuds. De la même façon, on pourrait expliquer l'absence de corrélation significative entre le nombre d'entre-nœuds et l'allongement moyen d'un entre-nœud. L'indépendance entre ces deux variables laisse supposer qu'il n'existe pas de phénomène de "compensation» comme il a été mis en évidence sur d'autres espèces.

2) Linfluence des précipitations de juin, juillet et septembre de l'année précédente, sur NE. Cléments (1970) sur Pinus resinosa note que la présence d'eau libre dans le sol pendant la phase d'initiation est plus importante que pendant la phase d'élongation. De même Sucoff (1971) toujours sur Pinus resinosa note que l'absence d'eau peut aller jusqu'à arrêter l'initiation.

Les années de meilleure croissance semblent être liées à la possibilité de continuer l'initiation à la fin de l'été et au début de l'automne. Kremer \& Roussel (1985) remarquent sur des provenances de pin maritime que la supériorité des provenances atlantiques est due à la fois à une plus grande durée d'initiation et à un plus fort taux de croissance absolu que chez les autres provenances.

3) Le rôle prépondérant des températures minimales sur l'allongement des entrenœuds. Les effets à court terme d'une baisse de la température moyenne journalière de l'air sur les taux de croissance ont été mentionnés par de nombreux auteurs dont Larson (1967), Boyer (1970) sur Pinus taeda, Odin H. (1972) sur pin sylvestre, Piétarinen et al. (1982). Aux deux années de plus faible élongation moyenne des entre-nœuds (1979 et 1984) correspondent les températures minimales les plus faibles de nos 6 années d'observation. Le rang de classement de 1981 est dû, pour la plus grande part, à un fort taux d'allongement coïncidant avec une température minimale élevée en début et au milieu de la période d'élongation. Cependant, un faible nombre d'entre-nœuds dont l'élongation serait importante ne saurait produire un accroissement total en hauteur identique à celui issu d'un grand nombre d'entre-nouds dont l'allongement moyen serait plus faible.

La prévision de la production en fonction des précipitations de juin, juillet et septembre de l'année précédente apporterait aux modèles de croissance du pin noir d'Autriche une partie des bases biologiques qui leur font actuellement défaut.

A terme, ce sont les relations indépendantes de la sylviculture (comme la liaison âge-hauteur) qui pourraient être améliorées en y intégrant un indice climatique qui tiendrait compte des précipitations pendant la période d'initiation et (ou) des températures minimales de la période d'élongation.

Nos mesures ne concernent que des jeunes peuplements, elles demandent donc à être complétées par des observations sur des peuplements adultes. De plus une étude en cours sur les taux d'initiation devrait permettre d'améliorer nos connaissances sur l'époque, la durée et l'intensité de ce mécanisme biologique.

\section{Reçu en janvier 1985. Accepté en juin 1985.}

\section{Remerciements}

Nous tenons à remercier tout particulièrement :

- MM. J. Toth et M. Turrel qui ont mis en place le dispositif expérimental et réalisé certaines mesures dendrométriques utilisées ici ;

- $\mathbf{M}^{m \prime}$ R. Cimapnls; qui a réalisé la frappe;

- M. J. BetTACHini qui a exécuté les graphiques;

enfin MM. Birot et Ducrey de la Station de Sylviculture méditerranécnne et A. KREMER du Laboratoire d'Amélioration des Arbres forestiers à Pierroton qui ont bien voulu relire le manuscrit et nous faire part de leurs remarques. 


\section{Summary \\ Effects of climatic factors on height growth components in Austrian black pine} (Pinus nigra ssp. nigricans Arn.)

Weekly (or biweekly) leader shoot growth was assessed over 3 growing seasons (1982-1984) and annual shoot length was measured over 6 years (1979-1984) for a 30 seedlings (age : 11 years) sampled in a naturally regenerated stand of black pine, located at Mont Ventoux in southern France.

The number of internodes (stem units) was assessed over the same period (1979-1984).

Annual shoot length of a given year appeared as mainly controlled by the number of internodes initiated during the summer of the previous year. Investigations of climatic factors taking place during the assumed initiation stage, have shown a significant correlation between the annual leader shoot length and the cummulated rainfall of June, July and September of the previous year. By contrast, the correlations between temperature factors during the assumed time of initiation and number of initiated internodes of the same year were not significant. The weckly shoot growth was significantly related to the average of minimum air temperature of the same period, at the time of height growth, that is to say April and May.

Finally the possibility of integrating these results into growth models is discussed.

Key words : Height growth, morphogenesis, morphogenetic cycle, growth rate, temperature, rainfall, Pinus nigra, cataphyll initiation, stem unit elongation.

\section{Références bibliographiques}

Boyer W.D., 1970. Shoot growth Patterns of yourg Loblolly Pine. For. Sci., 16 (4), 472-482.

Canneli. M.G.R., 1978. Component of conifer shoot growth. In Proceedings of the 5th North American forest biology workshop. Eds. Hollins C.A., Squillace A.E., 313-318, Univ. Flor. Gorinesville.

CAUSTON Dr., Vénus J.C., 1981. The biometry of plant growth. Edward Arnold, 307 p.

CLÉMENTS J.R., 1970 Shoots responses of young red pine to watering applied over two seasons. Can. J. Bot., 48, 75-80.

Debazac E.F., 1963. Morphologie et sexualité chez les pins. Rev. For. Fr., 14 (4), 213-303.

Garrett P.W., Zahner R., 1973. Fascicle density and needle growth responses of red pine to water supply over two seasons. Ecology, 54, 1328-1334.

Kremer A., 1981. Déterminisme génétique de la croissance en hauteur du pin maritime (Pinus Pinaster Ait.). I. Rôle du polycyclisme. Ann. Sci. For., 38 (2), 199-222.

Kremer A., 1981. Déterminisme génétique de la croissance en hauteur du pin maritime (Pinus Pinaster Ait.). II. Comportement interannuel. Interaction génotype $\mathrm{X}$ année. Ann. Sci. For., 38 (3), 331-355.

Kremer A., Larson P.R., 1983. Genetic control of height growth components in Jack pine seedlings. Forest sci., 29 (3), 451-464.

Kremer A., Roussel G., 1982. Composantes de la croissance en hauteur chez le pin maritime (Pinus pinaster Ait.). Ann. Sci. For., 39, 77-98.

Kremer A., Roussel G., 1985. Décomposition de la croissance en hauteur. Variabilité géographique des composantes morphogénétiques et phénologiques. A.S.F. (sous presse). 
LANNER R.M., 1968. The pine shoot primary growth system. Ph. D. Thesis, Univ. Minn, St-Paul, $136 \mathrm{p}$.

LARSON P.R., 1967. Effects of temperature on the growth and wood formation of tow pinus resinosa sources. Silvae Genetica, 16 (2), 58-65.

Lascoux D., 1984. Décomposition de la croissance en hauteur du pin maritime (Pinus pinaster Ait). Aspects morphogénétiques et génétiques. Mémoire de fin d'études à l'E.N.I.T.E.F., $81 \mathrm{p}$.

OdiN H., 1972. Studies of the increment rhythm of scots pine and Norway spruce plants. Studia Forestalia Suesica, 97, 31 p. Eds. Skogshogskolan Royal college of forestry Stockholm.

OWEns J.N., 1984. Bud development in Grand Fir (Abies grandis). Can. J. For. Res., 14, 575-588.

Pietarinen I., Kanninen M., Hari P., Kellomaki S., 1982. A simulation model for daily growth of shoots, needles, and stem diameter in Scots Pine trees. For. Sci., 28 (3), $573-581$.

Pollard D F.W., Logan K.T., 1977. The effects of light intensity, photoperiod, soil moisture potential and temperature on bud morphogenesis in Picea species. Can. J. For. Res., 7, 415-421.

Richards F.J., 1959. A flexible growth function for empirical use. J. Exp. Bot., 10, 290-300.

Sucoff E., 1971. Timing and rate of bud formation in Pinus Resinosa. Can. J. Bot., 49, 1821-1832.

Tотн J., 1984. (Communication personnelle). 